\title{
BMJ Open Strength of the association between Turner syndrome and coeliac disease: protocol for a systematic review and meta-analysis
}

\author{
Ghada S M Al-Bluwi (D , , ${ }^{1}$ Asma H Alnababteh, ${ }^{2}$ Saif Al-Shamsi (D),${ }^{1}$ \\ Rami H Al-Rifai (D) ${ }^{2}$
}

To cite: Al-Bluwi GSM, Alnababteh AH, Al-Shamsi S, et al. Strength of the association between Turner syndrome and coeliac disease: protocol for a systematic review and meta-analysis. BMJ Open 2020;10:e037478. doi:10.1136/ bmjopen-2020-037478

- Prepublication history and additional material for this paper are available online. To view these files, please visit the journal online (http://dx.doi. org/10.1136/bmjopen-2020037478).

Received 04 February 2020 Revised 03 June 2020 Accepted 04 June 2020

Check for updates

(c) Author(s) (or their employer(s)) 2020. Re-use permitted under CC BY-NC. No commercial re-use. See rights and permissions. Published by BMJ.

${ }^{1}$ Department of Internal Medicine, College of Medicine and Health Sciences, United Arab Emirates University, Al Ain, United Arab Emirates

${ }^{2}$ Institute of Public Health, College of Medicine and Health Sciences, United Arab Emirates University, Al Ain, United Arab Emirates

Correspondence to

Dr Rami H Al-Rifai;

rrifai@uaeu.ac.ae

\section{ABSTRACT}

Introduction Coeliac disease (CD) is a genetic autoimmune disorder characterised by a permanent sensitivity to the gluten contained in some grains. Certain patient groups are considered high risk for the development of $C D$, including, but not limited to, those with chromosomal disorders such as Turner syndrome (TS). Here, we present a protocol for a systematic review and meta-analysis that aims to comprehensively summarise the literature, and quantitatively estimate the weighted strength of the association between TS and CD.

Methods and analysis Our protocol follows the Preferred Reporting Items for Systematic Review and Meta-Analysis Protocols 2015 guidelines. We will search PubMed, Scopus, Web of Science and Embase databases for relevant articles. Variant and broad search terms will be selected for identifying epidemiological studies reporting on the crude and/or adjusted association between TS and $\mathrm{CD}$. Retrieved citations will be screened, and data from the eligible research reports against specific eligibility criteria will be extracted. We will then assess the risk of bias associated with the eligible studies using the NewcastleOttawa Scale. The overall weighted strength of the pooled association will be quantified using the random-effects model.

Ethics and dissemination This review will use data from published literature; hence, ethical approval will not be needed. The resulting review will be the first to produce a comprehensive synthesis of the strength of the association between TS and CD. The results will be disseminated through a peer-reviewed journal as well as in local and international conferences and symposiums. Results dissemination would help healthcare providers and policy-makers to make informed decisions regarding the diagnosis and management of $C D$ in high-risk individuals. PROSPERO registration number CRD42019131881, dated 3 September 2019.

\section{INTRODUCTION}

In recent decades, there has been a dramatic increase in the prevalence of coeliac disease (CD), worldwide. ${ }^{12} \mathrm{~A}$ recent meta-analysis found that the global prevalence of $\mathrm{CD}$ was $1.4 \%$ based on serological testing, and $0.7 \%$ based on small intestinal biopsy. According
Strengths and limitations of this study

- To ensure all relevant evidence is captured, this review will include all observational studies reporting an association between Turner syndrome and the development of coeliac disease and the review will be reported according to the Preferred Reporting Items for Systematic Review and Meta-Analysis Protocols 2009 guidelines.

- To have a highly sensitive and specific database search strategy, an expert librarian in designing search strings for systematic review and metaanalysis studies will be involved.

- Citations screening, eligible studies identification, data extraction and risk-of-bias assessment will be performed by at least two well-trained reviewers to ensure avoiding and minimising selection and data extraction bias to the lowest level possible.

- In the event of high heterogeneity in measures of association between Turner syndrome and coeliac disease, and contingent on the availability of enough estimates of association-the source of heterogeneity through subgroup as well as univariate and multivariate metaregression analyses will be explored; otherwise, the review might be reported narratively.

to that meta-analysis, the prevalence of CD varied by location. In South America, the prevalence of $\mathrm{CD}$ was $4.0 \%$; in Africa and North America, it was $0.5 \%$, in Asia $0.6 \%$ and in Europe and Oceania $0.8 \% .^{3}$

$\mathrm{CD}$ is a genetically based autoimmune disorder characterised by a permanent sensitivity to gluten-containing grains, such as wheat, barley and rye, and their derivatives. ${ }^{4}$ The key genetic factor associated with $\mathrm{CD}$ is the human leucocyte antigen (HLA) with more than $90 \%$ of patients with CD carrying either the HLA-DQ2 or HLA-DQ8 genotypes. ${ }^{5}$ The consumption of gluten or repeated gastrointestinal infections early in life in genetically susceptible individuals are 
believed to trigger and drive the induction of intraepithelial lymphocytes in the small intestine, leading to villous atrophy. ${ }^{6-8}$ The classical clinical manifestations of patients with CD are usually diarrhoea, weight loss or failure to thrive. However, they may present with nonclassical signs and symptoms, such as anaemia, abdominal pain, osteomalacia and osteoporosis, short stature, lymphoma, liver disease and neurological and psychological symptoms. ${ }^{9} 10$

Although screening for $\mathrm{CD}$ is not recommended for the general population currently, certain patient groups are considered high risk for the development of CD; screening is recommended for such groups. ${ }^{11}{ }^{12}$ Different high-risk groups have been identified by multiple studies; among these groups are first-degree relatives of patients with $\mathrm{CD}$, patients with immune-mediated conditions including type 1 diabetes mellitus and autoimmune thyroid disease or patients with selective IgA deficiency, and those with genetic disorders such as Down, Turner and Williams syndrome. ${ }^{13-15}$ A meta-analysis showed that $1.6 \%$ of patients with autoimmune thyroid disease have CD. ${ }^{16}$ Another meta-analysis revealed that around 1 in 31 patients with iron deficiency anaemia had histological evidence of $\mathrm{CD},{ }^{17}$ and approximately $2.0 \%$ of people with osteoporosis had biopsy-verified CD. ${ }^{18}$

Turner syndrome (TS) is a chromosomal disorder that affects around 1 in 2500 live female births. It is the most commonly observed $\mathrm{X}$ chromosome-related genetic disorder in females, and is characterised by short stature, ovarian dysfunction, cardiovascular disease and an increased risk of autoimmune diseases such as diabetes type 1 and $\mathrm{CD} .{ }^{19}$ Several studies have demonstrated that CD is more commonly observed in patients with TS than those in the general population. ${ }^{20-24}$ Therefore, it has been recommended that patients with TS be monitored for $\mathrm{CD}^{11}$

There is a lack of systematic reviews on the strength of association between specific genetic disorders and $\mathrm{CD} .{ }^{25}$ Estimating the pooled prevalence would reflect only the burden of disease. Furthermore, the prevalence of $\mathrm{CD}$ in cases of TS does not provide any information on whether TS increases the risk of CD and by how much. In contrast, a statistical measure of association between TS and the risk of developing CD provides this information directly. Moreover, producing a pooled measure of association would allow and minimise bias in estimating (1) the attributable risk fraction of TS in developing CD among individuals with TS and (2) the population attributable risk fraction of TS in developing CD among the entire population. However, the success of this approach is contingent on the availability of the prevalence estimates. The present protocol describes the methodology for a systematical literature review and quantitative estimation of the strength of the association between TS and $\mathrm{CD}$. The findings of the review will fill a gap in the literature, and may inform the planning and implementation of effective early screening and control programmes for CD among those with TS.
The systematic review that is to be conducted following this protocol aims to narratively summarise all the available literature, and quantify a weighted estimate of the strength of the association between TS and CD, from 1 January 1991 to 1 December 2019.

\section{Objectives}

The specific objectives of the systematic review are as follows:

1. To systematically review and narratively summarise literature reporting the association between TS and CD.

2. To quantitatively summarise the weighted crude and adjusted strength of the association between TS and $\mathrm{CD}$, in the form of relative risk (RR).

\section{Research question}

The present protocol for a systematic review and metaanalysis study will help in answering our research question: 'Are patients with TS at a higher risk of having CD than those without?'

\section{MATERIALS AND METHODS Protocol and registration}

This protocol adheres to the Preferred Reporting Items for Systematic Review and Meta-Analysis Protocols (PRISMA-P) 2015 statement (online supplementary table 1). ${ }^{26}$ The systematic review will be reported according to the PRISMA (2009) statement guidelines, ${ }^{27}$ and the Meta-analyses Of Observational Studies in Epidemiology checklist. $^{28}$

\section{Eligibility criteria}

The protocol has been developed in consideration of the participants, exposure, comparator, outcome (s), and type of study $(\mathrm{PECO}(\mathrm{T}))$. The PECO $(\mathrm{T})$ statement provides the framework from which studies are identified and selected for inclusion. ${ }^{29}$ However, as we are looking for observational studies, we only considered participants, exposure and the outcome of interest.

\section{Participants}

The participants included should be females with a TS diagnosis regardless of age.

\section{Exposure}

TS presence. All TS cases regardless of type (mosaic or classical).

\section{Outcome}

Our outcome of interest is $\mathrm{CD}$ regardless of the diagnostic criteria.

\section{Type of studies}

All epidemiological observational studies that report on the calculated association between TS and CD, or that allow for the calculation of the crude and/or adjusted estimate of the association between TS and CD will be included. 
Publication period

In 1990, The European Society of Gastroenterology, Hepatology and Nutrition released the first modern guidelines for CD diagnosis. ${ }^{30}$ Therefore, we will consider the year 1990 as the year for the well-defined CD diagnostic criteria. All relevant articles published from January 1991 to December 2019 will be included in this systematic review.

We will exclude all other studies not meeting our eligibility criteria.

\section{Data sources and search strategy}

Four major electronic databases, PubMed, Scopus, Web of Science and Embase, will be used to conduct a literature search to identify eligible publications. The reference lists of the eligible publications will be hand searched to identify further studies that may have been missed.

A comprehensive and sensitive computerised search will be implemented for identifying eligible published studies. We will use specific search terms including variant terms using Boolean operators. To ensure the comprehensiveness of the search strategy, and to avoid potential eligible studies being missed, a predetermined electronic search strategy will be designed by the search team (GSM-AB and S-AS) and a librarian with expertise in designing search terms for systematic reviews. Our designed search strategy is presented in the online supplementary box 1 .

\section{Identification of the eligible studies}

All the retrieved search citations will be imported to Covidence software (Covidence, Melbourne, Australia), ${ }^{31}$ where all duplicates will be removed automatically.

\section{Titles and abstract screening}

Of the remaining citations, the titles and abstracts will be screened for relevance by at least two independent reviewers. Screened titles and abstracts will be classified into three categories: not relevant, relevant or potentially relevant. A third reviewer will resolve articles that fall in the conflict zone.

\section{Full-text screening}

The full texts of the identified relevant or potentially relevant publications will be thoroughly screened, and independently assessed by at least two reviewers. Additional studies with potential duplicate estimates on the same study subjects will be removed during the fulltext screening. Discrepancies between reviewers will be resolved by a third reviewer.

\section{Data extraction}

Relevant data from eligible studies will be extracted into a predefined data extraction form, which will first be piloted using five eligible research reports. The data will be extracted independently by two review investigators. Discrepancies between data extractors will be discussed and resolved for a consensus to be reached. If a consensus cannot be achieved, an expert will be consulted.
The following parameters will be extracted from relevant studies: author names, year of publication, specific country and city in which the study was conducted, study design, setting, study period, CD diagnostic criteria used, type of TS, number of participants tested for CD, mean or range age of the tested study subjects, number of tested subjects found positive for $\mathrm{CD}$, frequency of screening, numbers of patients with and without TS diagnosed with $\mathrm{CD}$, crude and adjusted estimates of the association between TS and CD and their 95\% CIs. We will contact the relevant corresponding authors to obtain any missing data.

A complete list of the precoded parameters to be extracted is provided in online supplementary table 2.

\section{Quality and risk-of-bias assessment}

Two reviewers will independently assess the quality of the eligible studies. We will assess each study's risk of bias using the Newcastle-Ottawa Scale (NOS) for observational studies. ${ }^{32}$ The NOS will be adapted from its original version to make it specific to and suitable for this review.

\section{Data synthesis}

For studies providing an overall adjusted estimate of the association between TS and CD using several models, we will consider the estimate obtained from the model adjusted for the largest numbers of variables. For studies providing stratified crude estimates of the association between TS and CD, the estimate of the association of the total sample will be replaced with that of the pooled stratified measures. A predefined sequential order will be followed when considering stratified measures. Estimates stratified according to age will be prioritised, followed by countries and year. This scheme will prioritise strata with more information on the tested subjects. Otherwise, the overall estimate of the association will be included. One stratification level per included published research report will be considered to avoid double counting.

\section{Summary measures and synthesis of results: meta-analysis}

Using the random-effects model, we will perform metaanalyses of the extracted data for the estimation of the weighted pooled strength of the association between TS and CD and its corresponding 95\% CI. The strength of the association will be quantified in the form of RR and adjusted RR. Since we are including all observational studies regardless of the type of the measure of association between TS and CD, we are expecting a lack of uniformity in the reported measures of association in the literature. For instance, some studies might report an odds ratio (OR) while others might report RR. However, to obtain a unified and consistent measure of association across all studies, in the form of RR, all estimates in the form of OR will be converted into RR, following a standard procedure using the following mathematical formula. ${ }^{33}$ This will be done for crude and adjusted estimates separately

$$
\mathrm{RR}=\mathrm{OR} /\left(1 \mathrm{p}_{0}+\left(\mathrm{p}_{0} \times \mathrm{OR}\right)\right)^{3435}
$$


where $\mathrm{p}_{0}$ is the baseline risk that is calculated using the following formula:

Baseline risk $=\mathrm{OR} /(1+\mathrm{OR})$

We will use the metan command in Stata software (StataCorp) ${ }^{36}$ for the performance of a meta-analysis of the crude and adjusted estimates of association, separately. The metan command incorporates the FreemanTukey double arcsine transformation for the stabilisation of the variances of estimate measures.

As for the adjusted estimates, based on the reported confounders adjusted for in the individual studies, in the subgroup analyses, we will pool estimates together adjusted for the same confounders as long as enough data are available. For example, all estimates adjusted for age will be pooled in one meta-analysis model while those adjusted for age and other chronic diseases will be pooled using a separate meta-analysis model. This would allow us to produce more precise and informative estimates of association.

The effect estimates will be weighted using the inverse variance method. ${ }^{37}$ For each pooled estimate and its $95 \%$ CI, a forest plot will be created to show the effect estimate and corresponding 95\% CI for each study and the overall weighted effect estimate.

The heterogeneity in effect estimates will be evaluated across studies. Specifically, we will conduct Cochran's $\mathrm{Q}$ test and extract several heterogeneity measures, including the estimate of the between-study variance of the true effect sizes using the $\tau^{2}$ statistic, the magnitude and the $95 \%$ CI of the between-study variation attributed to heterogeneity rather than chance using the $\mathrm{I}^{2}$ statistic, and the $95 \%$ prediction interval that estimates the distribution of the true effect size among the included studies. ${ }^{38}$ The Q-statistic tests for heterogeneity are based on the null hypothesis that all studies share a common effect size. Hypothesis testing will be performed based on a $p$ value $<0.10$, implying that the studies do not share a common effect size. ${ }^{38}$

\section{Assessment of metabias}

To assess publication bias, we will examine funnel plots supplemented with formal statistical testing using Egger's test. ${ }^{40}$ A funnel plot of effect estimates will be plotted against the sample size. ${ }^{41}$ The asymmetry of the funnel plot will be examined by performing an Egger's test. ${ }^{40}$

\section{Sources of heterogeneity: metaregression}

Random-effects univariate models and multivariable metaregression models will be used for identifying sources of between-study heterogeneity and quantifying their contribution to variability in the effect estimates. In the univariate metaregression models, analyses will be performed based on tested population, study period, CD diagnostic criteria and sample size. All variables with a $p$ value $<0.1$ in the univariate models will be included in the multivariable model. In the final multivariable model, a $p$ value $\leq 0.05$ will be considered statistically significant, contributing to heterogeneity in the effect estimates.
All metaregression analyses will be performed using the metareg package in Stata/SE V.15. ${ }^{36}$

\section{Patient and public involvement}

No patients were involved in the design of this protocol or the study to be carried out.

\section{Ethics and dissemination}

This review will use data from published literature; hence, ethical approval is not needed. The results of this proposed systematic review will be disseminated to healthcare providers and policy-makers. The findings may aid in updating relevant guidelines, and help inform clinicians to make decisions regarding the diagnosis and management of $\mathrm{CD}$ in high-risk individuals.

Contributors GSMA-B, AHA and SA-S conceptualised the research question. All authors contributed equally to protocol drafting and writing. RHA-R designed the analysis plan, critically revised the protocol and approved the final version. All authors read and approved the final manuscript. RHA-R and SA-S are the guarantors of the review.

Funding The authors have not declared a specific grant for this research from any funding agency in the public, commercial or not-for-profit sectors.

Competing interests None declared.

Patient and public involvement Patients and/or the public were not involved in the design, or conduct, or reporting, or dissemination plans of this research.

Patient consent for publication Not required.

Provenance and peer review Not commissioned; externally peer reviewed.

Open access This is an open access article distributed in accordance with the Creative Commons Attribution Non Commercial (CC BY-NC 4.0) license, which permits others to distribute, remix, adapt, build upon this work non-commercially, and license their derivative works on different terms, provided the original work is properly cited, appropriate credit is given, any changes made indicated, and the use is non-commercial. See: http://creativecommons.org/licenses/by-nc/4.0/.

\section{ORCID iDs}

Ghada S M Al-Bluwi http://orcid.org/0000-0002-5850-7002

Saif Al-Shamsi http://orcid.org/0000-0001-9755-3493

Rami H Al-Rifai http://orcid.org/0000-0001-6102-0353

\section{REFERENCES}

1 Rubio-Tapia A, Kyle RA, Kaplan EL, et al. Increased prevalence and mortality in undiagnosed celiac disease. Gastroenterology 2009;137:88-93.

2 Lohi S, Mustalahti K, Kaukinen K, et al. Increasing prevalence of coeliac disease over time. Aliment Pharmacol Ther 2007;26:1217-25.

3 Singh P, Arora A, Strand TA, et al. Global Prevalence of Celiac Disease: Systematic Review and Meta-analysis. Clin Gastroenterol Hepatol 2018;16:823-36.

4 Guandalini S, Assiri A. Celiac disease: a review. JAMA Pediatr 2014;168:272-8.

5 Andrén Aronsson C, Kurppa K, Agardh D. Gluten in infants and celiac disease risk. Expert Rev Gastroenterol Hepatol 2016;10:669-70.

6 Sollid LM, Jabri B. Triggers and drivers of autoimmunity: lessons from coeliac disease. Nat Rev Immunol 2013;13:294-302.

7 Kemppainen KM, Lynch KF, Liu E, et al. Factors that increase risk of celiac disease autoimmunity after a gastrointestinal infection in early life. Clin Gastroenterol Hepatol 2017;15:694-702.

8 Green PHR, Cellier C, disease C. Celiac disease.. N Engl J Med 2007;357:1731-43.

9 Ludvigsson JF, Bai JC, Biagi F, et al. Diagnosis and management of adult coeliac disease: guidelines from the British Society of gastroenterology. Gut 2014;63:1210-28.

10 Laurikka P, Nurminen S, Kivelä L, et al. Extraintestinal manifestations of celiac disease: early detection for better long-term outcomes. Nutrients 2018;10. doi:10.3390/nu10081015. [Epub ahead of print: 03 Aug 2018]. 
11 Aggarwal S, Lebwohl B, Green PHR. Screening for celiac disease in average-risk and high-risk populations. Therap Adv Gastroenterol 2012;5:37-47.

12 Mearin ML. The prevention of coeliac disease. Best Pract Res Clin Gastroenterol 2015;29:493-501.

13 Hill ID, Dirks MH, Liptak GS, et al. Guideline for the diagnosis and treatment of celiac disease in children: recommendations of the North American Society for pediatric gastroenterology, hepatology and nutrition. J Pediatr Gastroenterol Nutr 2005;40:1-19.

14 Richey R, Howdle P, Shaw E, et al. Recognition and assessment of coeliac disease in children and adults: summary of NICE guidance. BMJ 2009;338:b1684.

15 Husby S, Koletzko S, Korponay-Szabó IR, et al. European Society for pediatric gastroenterology, hepatology, and nutrition guidelines for the diagnosis of coeliac disease. J Pediatr Gastroenterol Nutr 2012;54:136-60.

16 Roy A, Laszkowska M, Sundström J, et al. Prevalence of celiac disease in patients with autoimmune thyroid disease: a metaanalysis. Thyroid 2016;26:880-90

17 Mahadev S, Laszkowska M, Sundström J, et al. Prevalence of celiac disease in patients with iron deficiency Anemia-A systematic review with meta-analysis. Gastroenterology 2018;155:374-82.

18 Laszkowska M, Mahadev S, Sundström J, et al. Systematic review with meta-analysis: the prevalence of coeliac disease in patients with osteoporosis. Aliment Pharmacol Ther 2018;48:590-7.

19 Goldacre MJ, Wotton CJ, Seagroatt V, et al. Cancers and immune related diseases associated with Down's syndrome: a record linkage study. Arch Dis Child 2004;89:1014-7.

20 Marild K, Stordal K, Hagman A, et al. Turner syndrome and celiac disease: a case-control study. Pediatrics 2016;137: e20152232.

21 Bonamico M, Bottaro G, Pasquino AM, et al. Celiac disease and Turner syndrome. J Pediatr Gastroenterol Nutr 1998;26:496-9.

22 Jørgensen KT, Rostgaard K, Bache I, et al. Autoimmune diseases in women with Turner's syndrome. Arthritis Rheum 2010;62: 658-66.

23 Goldacre MJ, Seminog OO. Turner syndrome and autoimmune diseases: record-linkage study. Arch Dis Child 2014;99:71-3.

24 Mårild K, Størdal K, Hagman A, et al. Turner syndrome and celiac disease: a case-control study. Pediatrics 2016;137:e20152232.

25 Du Y, Shan L-F, Cao Z-Z, et al. Prevalence of celiac disease in patients with Down syndrome: a meta-analysis. Oncotarget 2018:9:5387-96.
26 Shamseer L, Moher D, Clarke M, et al. Preferred reporting items for systematic review and meta-analysis protocols (PRISMA-P) 2015: elaboration and explanation. BMJ 2015;350:g7647.

27 Moher D, Shamseer L, Clarke M, et al. Preferred reporting items for systematic review and meta-analysis protocols (PRISMA-P) 2015 statement. Syst Rev 2015;4:1.

28 Stroup DF, Berlin JA, Morton SC, et al. Meta-Analysis of observational studies in epidemiology: a proposal for reporting. meta-analysis of observational studies in epidemiology (moose) group. JAMA 2000;283:2008-12.

29 Woodruff TJ, Sutton P. The navigation guide systematic review methodology: a rigorous and transparent method for translating environmental health science into better health outcomes. Environ Health Perspect 2014;122:1007-14.

30 Revised criteria for diagnosis of coeliac disease. Report of working group of European Society of paediatric gastroenterology and nutrition. Arch Dis Child 1990;65:909-11.

31 Covidence. Covidence - better systematic review management. Melbourne, Victoria, Australia: Covidence, 2018. https://www. covidence.org/home

32 Wells GSB, O'Connell D, et al. The Newcastle-Ottawa scale (NOS) for assessing the quality of nonrandomised studies in meta-analysis, 2000.

33 Grant RL. Converting an odds ratio to a range of plausible relative risks for better communication of research findings. BMJ 2014;348:f7450.

34 Prasad K, Jaeschke R, Wyer P, et al. Tips for teachers of evidencebased medicine: understanding odds ratios and their relationship to risk ratios. J Gen Intern Med 2008;23:635-40.

35 Shrier I, Steele R. Understanding the relationship between risks and odds ratios. Clin J Sport Med 2006;16:107-10.

36 StataCorp. Stata statistical software: release 15. College Station TSL.

37 Miller JJ. The inverse of the Freeman-Tukey double arcsine transformation. Am Stat 1978;32:138

38 Higgins JPT, Thompson SG, Spiegelhalter DJ. A re-evaluation of random-effects meta-analysis. J R Stat Soc Ser A Stat Soc 2009;172:137-59.

39 Borenstein M, Hedges LV, Higgins JPT, et al. Introduction to metaanalysis. Wiley, 2009.

40 Egger M, Smith GD. Meta-Analysis. potentials and promise. BMJ 1997;315:1371-4.

41 Hunter JP, Saratzis A, Sutton AJ, et al. In meta-analyses of proportion studies, funnel plots were found to be an inaccurate method of assessing publication bias. J Clin Epidemiol 2014;67:897-903. 